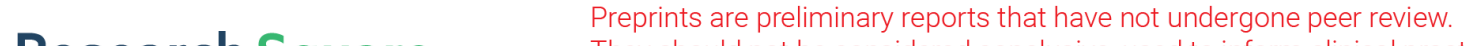 They should not be considered conclusive, used to inform clinical practice, or referenced by the media as validated information. \\ Identification of key genes in relapsed multiple myeloma by weighted gene co-expression network analysis
}

\section{Lele Cao'}

Second Affiliated Hospital of Anhui Medical University

\section{Ye Meng'}

Second Affiliated Hospital of Anhui Medical University

\section{Rui Huang}

Second Affiliated Hospital of Anhui Medical University

Xiaoxue Wang

Second Affiliated Hospital of Anhui Medical University

Hui Qin ( $\nabla$ qinhui6957@sina.com )

Second Affiliated Hospital of Anhui Medical University https://orcid.org/0000-0002-2242-8257

\section{Research}

Keywords: multiple myeloma, relapse, weighted gene co-expression network, key genes

Posted Date: January 25th, 2021

DOI: https://doi.org/10.21203/rs.3.rs-153300/v1

License: (c) (i) This work is licensed under a Creative Commons Attribution 4.0 International License. Read Full License 


\section{Abstract \\ Background}

Multiple myeloma is a hematologic disorder of abnormal plasma cell proliferation. Although there are some agents with different mechanisms in the clinic, the treatment of multiple myeloma is still challenging for the reason that its recurrence and progression are common. Therefore, it is critical to determine novel biomarkers to improve the prognosis of patients.

\section{Methods}

Firstly, raw data of GSE82307 was collected from the Gene Expression Omnibus database. Secondly, the top $50 \%$ of most variant genes were employed to construct a gene co-expression network in the R.WGCNA algorithm, and module significance and module membership were utilized to identify hub modules and hub genes respectively. The gene ontology enrichment and Kyoto encyclopedia of genes and genomes pathway analysis were carried out to assess biological characteristics. Then a protein-protein interaction network was conducted based on the STRING website and Cytoscape software. Next, differentially expressed genes were analyzed using the limma R package. Finally, survival analysis was performed by Kaplan-Meier plotter to evaluate prognosis.

\section{Results}

10826 genes were used to construct a co-expression network. In this network, the blue module was identified as a hub module in which 68 genes were identified as hub genes. Furthermore, 46 differentially expressed genes were screened in samples of GSE82307. Integrating hub genes and differentially expressed genes, we determined 14 key genes. Finally, survival analysis revealed that ten genes CDCA5, CEP55, HJURP, CDC20, FOXM1, RRM2, TTK, CENPE, SKA1, NUF2 were related to the relapse and prognosis of multiple myeloma.

\section{Conclusion}

Our study suggested that CDCA5, CEP55, HJURP, CDC20, FOXM1, RRM2, TTK, CENPE, SKA1, NUF2 may be potential biomarkers for predicting the relapse and prognosis of multiple myeloma.

\section{Introduction}

Multiple myeloma(MM) is a systemic hematological malignancy that is characterized by the uncontrolled growth of monoclonal plasma cells in the bone marrow. The main clinical manifestations of $\mathrm{MM}$ are osteolytic lesions, hypercalcemia, anemia, and kidney damage. MM is the second most common hematologic malignancy, second only to non-Hodgkin's lymphoma, accounting for $13 \%[1]$. And its median 
age of onset is $63 \sim 70$ years old[2]. In the past 20 years, the application of autologous stem cell transplantation after high-dose chemotherapy, immunomodulator(IMiD), proteasome inhibitor has significantly improved the prognosis of patients[3-5]. Nonetheless, MM is still an incurable biologically heterogeneous disease of plasma cells, and most patients will eventually relapse or progress. When a patient newly develops hypercalcemia, kidney damage, worsening anemia, bone damage, extramedullary disease, or a 'significant' biochemical relapse, we consider that this patient is in a state of relapse[6]. The causes of the recurrence of MM have also aroused widespread concern. Recurrence is the result of multiple factors in myeloma. These factors mainly contain acquired drug resistance and continued acceleration of tumor cell proliferation which are caused by additional genetic mutations or genetic changes[7]. Therefore, the remission time after each salvage treatment is gradually shortened, eventually developing into relapsed MM. However, there is a lack of biomarkers for recurrence or progression of myeloma. Revealing candidate targets will provide tremendous help to the diagnosis, treatment, and prognosis of myeloma patients.

A large amount of genomic information is accumulated exponentially, with the continuous advancement of biological research techniques, especially the gradual development of sequencing technology and biological information algorithms[8, 9]. Weighted gene co-expression network analysis(WGCNA) is a systematic biological approach for appraising gene association modes among different samples[10]. WGCNA has been successfully used not only to explore the connection between genetic databases and clinical characterization but also to identify candidate biomarkers. However, the genetic difference between stable and relapsed MM patients remains unclear. Moreover, we need new biomarkers to better understand mechanisms of MM relapse and predict prognosis. Besides, Gene Expression Omnibus(GEO) is a public functional genomic database composing of an array- and sequence-based data. Therefore, we collected a GSE82307 gene expression profile submitted by Weinhold $\mathrm{N}$ et al[11] from GEO( http://www.ncbi.nlm.nih.gov/geo). Furthermore, we elucidated correlation patterns between genes through WGCNA-based systematic biology methods and identified new biomarkers related to relapsed MM.

\section{Methods}

\subsection{Acquisition of microarray data}

GSE82307 is an array- and sequence-based data profile dependent on the GPL570 platform(Affymetrix Human Genome U133 Plus 2.0 Array) including 66 gene expression datasets from 33 patients' samples collected at presentation and relapse respectively. By employing Affy and Limma packages in Bioconductor(http://www.bioconductor.org) in R software to read the array spectrum and standardize, as well as using the Robust Multi-Array Average algorithm, we acquired 21,653 genes for subsequent analysis.

\subsection{Construction of co-expression network}


According to variance analysis, the top 50\% (10826 genes) of most mutated genes were selected for further WGCNA. We utilized the WGCNA package to build a co-expression network of 10826 genes in $\mathrm{R}$ software. The objective of the co-expression network was to reveal the correlation between genes and to identify highly relevant gene modules. Firstly, the Pearson correlation matrix was constructed by using the absolute value of the correlation coefficient between genes. Then we used the power function to construct a weighted adjacency matrix. An appropriate soft threshold power $\beta$ was selected to convert the adjacency matrix to a topological overlap matrix. At last, we employed hierarchical clustering to generate a hierarchical clustering tree of genes.

\subsection{Identification of hub modules and hub genes}

Gene significance referred to the absolute value of the correlation between a gene and a clinical feature[12]. The module significance referred to the average value of gene significance of all genes in a module. These related values were displayed within a heatmap. Statistically significant modules were defined as $p$-value $<0.05$. In WGCNA, a module with the highest module significance score was generally defined as a hub module and chosen for further analysis $[10,13]$.

Hub genes were defined as genes with the highest correlation with clinical characteristics in a hub module. Module membership referred to the correlation between module eigengenes and gene expression profile[12]. Measured by Pearson correlation, the module membership score of hub genes was considered to be higher.

\subsection{Analysis of functional enrichment and pathway}

To better explore the biological significance of genes in hub modules, enrichment of functions and pathways was analyzed using the cluster profiler. Cluster profiler is a package with an analysis and visualization function to provide valuable information on gene ontology(GO) enrichment and Kyoto encyclopedia of genes and genomes(KEGG) pathway analyses[14, 15]. Uploading gene list of hub modules, we obtained results of biological process(BP), cellular component(CC), molecular function(MF), as well as KEGG pathway enrichment analysis. A p-value $<0.05$ was considered to be significantly enriched.

\subsection{Construction of protein-protein interaction (PPI) network}

After uploading hub genes to Interaction Gene/Protein Search Tool (STRING) online database, a network of correlation between proteins encoded by hub genes was constructed.

\subsection{Screening of differentially expressed genes(DEGs)}

Using the Limma R package[16], we filtered DEGs between $33 \mathrm{MM}$ patients at two treatment time points in GSE82307. Genes with |log2 Fold Change|value $>1$ and adj.P.Value $<0.05$ were defined as DEGs.

\subsection{Survival analysis}


The key genes are cross genes of hub genes obtained by the WGCNA module and DEGs. To further investigate the prognostic value of key genes for MM, we performed Kaplan-Meier curves of key genes by the $\mathrm{R}$ survival package. A $\mathrm{p}$-value $<0.05$ was considered to be statistically significant.

\section{Results}

\subsection{Construction of co-expression network and identification of hub modules and hub genes}

The appropriate soft threshold power was chosen to be 9 , which was based on the criterion of an approximate scale-free topology fit index 0.93(Fig. 1). We applied the R package of "WGCNA" to construct a weighted co-expression network. A total of 27 modules were identified, and the gene clustering was displayed as a dendrogram in Fig. 2. The identification of hub modules was based on module significance. Modules with the highest module significance score were usually defined as hub modules. According to the analysis of the module-feature relationship, the blue module(score.ModuleSignificance $=0.37, p=0.003)$ had the strongest correlation with recurrent MM(Fig. 3). Furthermore, 68 highly connected genes in the blue module were recognized as representative genes exhibiting potential critical functions in relapsed MM (score.ModuleMembership > 0.8, score.GeneSignificance >0.2)(Fig. 4).

\subsection{Analysis of functional enrichment and pathway and construction of PPI network}

We performed GO enrichment and KEGG pathway analysis to comprehend the biological characteristics of the above-mentioned 68 genes. A p-value $<0.05$ was considered to be significantly enriched. The top ten GO enrichment and KEGG pathway analysis were enumerated below(Fig. 5). In the GO enrichment analysis, 68 genes were mainly concentrated on organelle fission, chromosomal region, and catalytic activity, acting on DNA. In the KEGG pathway analysis, these genes were mainly enriched in the cell cycle, Human T-cell leukemia virus 1 infection, and DNA replication pathways. Through these dramatically rich biological functions and pathways, we can further investigate the effects of these genes on MM.

Then we uploaded 68 genes to Interaction Gene/Protein Search Tool(STRING) online database and utilized Cytoscape software to structure a PPI network for further analysis. The relationship network between proteins encoded by 68 hub genes was shown in the figure below(Fig. 6).

\subsection{Screening of DEGs}

We employed the R software package and limma package to filter out DEGs in $33 \mathrm{MM}$ patients at presentation and relapse in GSE82307(Fig. 7A). With a threshold at $\| \log 2$ Fold Changel $>1$ and adj.P.Value $<0.05$, a total of 46 DEGs were screened and consisted of 16 down-regulated genes and 30 up-regulated genes(Fig. 7B).

\subsection{Identification of key genes and survival analysis}


WGCNA identified 68 hub genes. Subsequently, DEGs analysis revealed 46 DEGs in 33 MM patients at presentation and relapse. In order to better capture valuable clues from these results, key genes were defined as cross genes of hub genes obtained by the WGCNA module and DEGs. Among a total of 97 genes, 14 genes BIRC5, CDCA5, CENPE, RRM2, TTK, FOXM1, HJURP, CEP55, PBK, UHRF1, SKA1, NUF2, CDC20, TACC3 were filtered out as key genes(Fig. 8). To further investigate the influences of key genes on overall survival(OS) and event-free survival(EFS) of MM, we adopted a Kaplan-Meier plotter for prognostic analysis(with a P-value $₫ 0.05$ ). As a result, patients whose tissues revealed a lower expression of CDC20, CDCA5, CENPE, CEP55, FOXM1, HJURP, NUF2, RRM2, SKA1, TTK showed significantly both longer OS and EFS compared with those with a higher expression(Fig. 9). To sum it up, we considered that the abovementioned ten key genes could serve as recurrence and prognosis biomarkers of MM.

\section{Discussion}

Nearly all myeloma patients eventually relapse, even those who experienced a complete response to previous treatment[17]. Therefore, clarifying underlying mechanisms that result in disease recrudesce may turn on new therapeutic approaches for MM. In the study, we firstly constructed a co-expression network of 10826 genes by the WGCNA algorithm based on GSE82307. Through analyzing module significance, module membership, and gene significance, 68 genes were recognized as representative hub genes in the blue hub module. Then performing GO enrichment and KEGG pathway analysis and constructing a PPI network, we comprehended the biological characteristics of hub genes and the relationship among proteins expressed by hub genes. Furthermore, a total of 46 DEGs including 16 downregulated genes and 30 up-regulated genes were screened in $33 \mathrm{MM}$ patients. At last, 14 cross genes BIRC5, CDCA5, CENPE, RRM2, TTK, FOXM1, HJURP, CEP55, PBK, UHRF1, SKA1, NUF2, CDC20, TACC3 of hub genes obtained by WGCNA and DEGs were filtered out as key genes. Survival analysis indicated patients with a lower expression of CDC20, CDCA5, CENPE, CEP55, FOXM1, HJURP, NUF2, RRM2, SKA1, TTK showed significantly both longer OS and EFS. Thus, the ten key genes might be potential biomarkers for the diagnosis and treatment of MM. The three genes CDCA5, CEP55, HJURP are usually overexpressed in several cancers and strongly correlated with the poor prognosis of patients. Notably, there is no relevant research on the effects of the three genes on MM so far. Apart from that, the remaining seven genes have been studied to varying degrees in $\mathrm{MM}$.

CDCA5 encodes sororin which is a regulatory protein during mitosis of eukaryotic cells. Sororin is pivotal for embryo development, maintenance of cohesion between sister chromatids, and correct chromosomal separation[18]. Sororin gene CDCA5 has been associated with the development and progression of several types of human cancers. In hepatocellular carcinoma, knockdown of CDCA5 by lentivirusmediated shRNA resulted in cell cycle arrest at the G2/M phase and cell apoptosis [19]. Moreover, higher expression of CDCA5 in hepatocellular carcinoma was associated with increased tumor size, microvascular infiltration, as well as poor prognosis of OS and DFS[20, 21]. Likewise, CDCA5 knockdown could inhibit the growth of esophageal squamous cell carcinoma cells. And the expression of CDCA5 promoted tumor cell proliferation, distant metastasis, drug resistance. The high expression of CDCA5 was related to tumor progression and shorter OS in esophageal squamous cell carcinoma[22]. Guanghou Fu et al. found that overexpression of CDCA5 in UMUC3 cells(bladder cancer cell line) could hasten cell 
proliferation and hinder cell apoptosis[23]. In an additional experiment, they also found overexpression of CDCA5 in bladder cancer cells activated PI3K/AKT pathway to promote cell proliferation and inhibited mitochondrial-mediated cell apoptosis through reducing expression of downstream proteins, such as cleaved caspase-3, caspase-9, and cleaved PARP. The results of our study demonstrated that CDCA5 was up-regulated in relapsed MM. And a higher expression of CDCA5 heralded a poor prognosis.

CEP55 is a centrosome- and midbody-associated protein and a key regulator of cytokinesis. Overexpressed CEP55 promotes proliferation, survival, and metastasis of multiple cancer cells at several levels[24]. Some characteristic components of CEP55 are also present in cell cycle and proliferation genes whose overexpression is also detrimental to clinical outcome[24]. CEP55 has been studied in the context of cancers including breast cancer, hepatocellular carcinoma, osteosarcoma et al. In breast cancer, the high CEP55 expression blocked cancer cell apoptosis and contributed to chemotherapy resistance, associated with genomic instability. For breast tumors exhibiting high CEP55 expression, blocking MEK1/2-PLK1 signaling reduced outgrowth of tumors[25]. In hepatocellular carcinoma, CEP55 promoted activation of the JAK2/STAT3 signaling pathway and induced expression of matrix metalloproteinases via the same signaling, which led to disease deterioration. Moreover, CEP55 was highly expressed in hepatocellular carcinoma cells, which heralded a poor prognosis[26]. In osteosarcoma, CEP55 was significantly overexpressed in tumor tissues, which was associated with tumor size, tumor metastasis, and reduced OS. Meanwhile, knockdown of CEP55 inhibited cell proliferation, invasion, and migration[27]. In the data set GSE82307, CEP55 was identified as one of the key genes related to relapsed MM, and the ROC curve indicated that CEP55 was a sign of poor prognosis for MM.

HJURP is a histone chaperone of nucleosomes that plays a dual role in coordinating the recruitment of CENP-A and CENP-C. HJURP assists in the assembly of functional centromeres and mediates chromosome separation and cell division[28]. The dysfunction of HJURP has been confirmed in kind of cancers. HJURP was highly expressed in liver cancer cells and tissues, which was a sign of poor prognosis. The high expression of HJURP promoted the proliferation of liver cancer cells by downregulating p21 through MAPK/ERK1/2 and AKT/GSK3 $\beta$ pathways[29]. Besides, the up-regulation of HJURP enhanced migration and invasion of liver cancer cells by interacting with sphingosine kinase 1[30]. In glioblastoma, HJURP knockout caused severe clonal ability and survival damage of glioblastoma cell lines. At the same time, these cell lines became more sensitive to radiation therapy after HJURP was reduced[31]. Moreover, HJURP was highly expressed in cells and tissues of pancreatic ductal cell carcinoma. HJURP may contribute to tumor cell growth, migration, invasion, and metastasis via the MDM2/p53 signaling pathway in pancreatic ductal cell carcinoma[32].

In order to further verify the carcinogenic ability and molecular roles of CDCA5, CEP55, HJURP in MM, we will continue to conduct in vivo, in vitro, and clinical research. The remaining seven genes CDC20, FOXM1, RRM2, TTK, CENPE, SKA1, NUF2 have been researched in MM to different depths. In the high-risk group of MM patients, the expression of CDC20 was significantly increased, accompanied by a shorter os and enrichment of genes related to proliferation. Besides, CDC20 knockout in myeloma cell lines could 
weaken cell apoptosis[33]. In MM patients showing high FOXM1 expression, both their DFS and OS were reduced. In vivo and in vitro experiments, down-regulation of FOXM1 inhibited the growth of myeloma cells, while up-regulation of FOXM1 resulted in the opposite[34]. RRM2 was significantly up-regulated. In terms of possible mechanisms, RRM2 participated in the proliferation and apoptosis of MM cells through the Wnt/ $\beta$-catenin signaling pathway[35]. TTK was a kinase related to the prognosis of MM, and its expression was associated with the high-risk group of MM. Besides, TTK inhibitors could significantly undermine the viability and proliferation capacity of myeloma cell lines and could be combined with Melphalan or IMiD as a new treatment strategy[36]. CENPE and SKA1 were upregulated in side population cells of MM, encoding for proteins associated with cell cycle and mitosis[37]. At last, during the culture of the fresh bone marrow of myeloma, down-regulation of NUF2 expression regulating mitosis and transcription was observed, which could reveal ensuing proliferation arrest[38]. However, the three genes CENPE, SKA1, NUF2 are initially found in MM. And their roles remain to be fully elucidated.

In summary, we adopted the WGCNA analysis to determine ten novel biomarkers CDCA5, CEP55, HJURP, CDC20, FOXM1, RRM2, TTK, CENPE, SKA1, NUF2 for recurrence and prognosis of MM. The results are beneficial to shed light on molecular mechanisms of progression and filter out targeted agents.

\section{Abbreviations}

MM: Multiple myeloma; IMiD: immunomodulator; WGCNA: Weighted gene co-expression network analysis; GEO: Gene Expression Omnibus; GO: gene ontology; KEGG: Kyoto encyclopedia of genes and genomes; BP: biological process; CC: cellular component; MF: molecular function; PPI: protein-protein interaction; DEGs: differentially expressed genes; OS: overall survival; EFS: event-free survival.

\section{Declarations}

\section{Acknowledgment}

We appreciate the GEO database(GSE82307) for free use.

\section{Authors' contributions}

LC and YM drew related charts and co-wrote this article. $\mathrm{RH}$ and $\mathrm{XW}$ collected biological data. HQ participated in the modifying and proofreading of this article. All authors read and approved the final manuscript.

\section{Funding}

No funding was obtained for this study.

\section{Availability of data and materials}


The profiles of GSE82307 can download in GEO datasets(https://www.ncbi.nlm.nih.gov/geo/query/acc.cgi?acc=GSE82307). The algorithm can be obtained by the corresponding author Hui Qin via Email (qinhui6957@sina.com).

\section{Ethics approval and consent to participate}

Not applicable.

\section{Consent for publication}

Not applicable.

\section{Competing interests}

The authors declare that they have no competing interests.

\section{Authors' information}

LC, YM, RH, XW, and HQ all work at the Department of Hematology, Second Affiliated Hospital of Anhui Medical University, Anhui Medical University, 678 Furong Road, Hefei 230601, Anhui, China.

\section{References}

1. Siegel RL, Miller KD, Jemal A. Cancer statistics, 2018. CA Cancer J Clin.2018;68:7-30.

2. Brenner $\mathrm{H}$, Gondos $A$, Pulte $D$. Recent major improvement in long-term survival of younger patients with multiple myeloma. Blood.2008;111:2521-2526.

3. Gonsalves WI, Rajkumar SV, Go RS, Dispenzieri A, Gupta V, Singh PP, Buadi FK, Lacy MQ, Kapoor P, Dingli $\mathrm{D}$ et al. Trends in survival of patients with primary plasma cell leukemia: a population-based analysis. Blood.2014;124:907-912.

4. Kumar SK, Dispenzieri A, Lacy MQ, Gertz MA, Buadi FK, Pandey S, Kapoor P, Dingli D, Hayman SR, Leung $\mathrm{N}$ et al. Continued improvement in survival in multiple myeloma: changes in early mortality and outcomes in older patients. Leukemia.2013;28:1122-1128.

5. Kumar SK, Rajkumar SV, Dispenzieri A, Lacy MQ, Hayman SR, Buadi FK, Zeldenrust SR, Dingli D, Russell SJ, Lust JA et al. Improved survival in multiple myeloma and the impact of novel therapies. Blood.2008;111:2516-2520.

6. Chim CS, Kumar SK, Orlowski RZ, Cook G, Richardson PG, Gertz MA, Giralt S, Mateos MV, Leleu X, Anderson KC. Management of relapsed and refractory multiple myeloma: novel agents, antibodies, immunotherapies and beyond. Leukemia.2018;32:252-262.

7. Egan JB, Shi CX, Tembe W, Christoforides A, Kurdoglu A, Sinari S, Middha S, Asmann Y, Schmidt J, Braggio $\mathrm{E}$ et al. Whole-genome sequencing of multiple myeloma from diagnosis to plasma cell leukemia reveals genomic initiating events, evolution, and clonal tides. Blood.2012;120:1060-1066. 
8. Orton RJ, Gu Q, Hughes J, Maabar M, Modha S, Vattipally SB, Wilkie GS. Bioinformatics tools for analysing viral genomic data. Revue Scientifique et Technique de l'OIE.2016;35:271-285.

9. Chen J, Coppola G. Bioinformatics and genomic databases. Handb Clin Neurol.2018;147:75-92.

10. Langfelder P, Horvath S. WGCNA: an R package for weighted correlation network analysis. BMC Bioinformatics.2008;9:559.

11. Weinhold N, Ashby C, Rasche L, Chavan SS, Stein C, Stephens OW, Tytarenko R, Bauer MA, Meissner T, Deshpande $S$ et al. Clonal selection and double-hit events involving tumor suppressor genes underlie relapse in myeloma. Blood.2016;128:1735-1744.

12. Horvath S, Dong J. Geometric interpretation of gene coexpression network analysis. PLoS Comput Biol.2008;4:e1000117.

13. Wright RM, Aglyamova GV, Meyer E, Matz MV. Gene expression associated with white syndromes in a reef building coral, Acropora hyacinthus. BMC Genomics.2015;16:371.

14. Huang da W, Sherman BT, Lempicki RA. Bioinformatics enrichment tools: paths toward the comprehensive functional analysis of large gene lists. Nucleic Acids Res.2009;37:1-13.

15. Huang da W, Sherman BT, Lempicki RA. Systematic and integrative analysis of large gene lists using DAVID bioinformatics resources. Nat Protoc.2009;4:44-57.

16. Ritchie ME, Phipson B, Wu D, Hu Y, Law CW, Shi W, Smyth GK. limma powers differential expression analyses for RNA-sequencing and microarray studies. Nucleic Acids Res.2015;43:e47.

17. Palumbo A, Anderson K. Multiple myeloma. N Engl J Med.2011;364:1046-1060.

18. Ladurner R, Kreidl E, Ivanov MP, Ekker H, Idarraga-Amado MH, Busslinger GA, Wutz G, Cisneros DA, Peters JM. Sororin actively maintains sister chromatid cohesion. Embo j.2016;35:635-653.

19. Shen Z, Yu X, Zheng Y, Lai X, Li J, Hong Y, Zhang H, Chen C, Su Z, Guo R. CDCA5 regulates proliferation in hepatocellular carcinoma and has potential as a negative prognostic marker. Onco Targets Ther.2018;11:891-901.

20. Hou S, Chen X, Li M, Huang X, Liao H, Tian B. Higher expression of cell division cycle-associated protein 5 predicts poorer survival outcomes in hepatocellular carcinoma. Aging (Albany NY).2020;12:14542-14555.

21. Tian Y, Wu J, Chagas C, Du Y, Lyu H, He Y, Qi S, Peng Y, Hu J. CDCA5 overexpression is an Indicator of poor prognosis in patients with hepatocellular carcinoma (HCC). BMC Cancer.2018;18:1187.

22. Xu J, Zhu C, Yu Y, Wu W, Cao J, Li Z, Dai J, Wang C, Tang Y, Zhu Q et al. Systematic cancer-testis gene expression analysis identified CDCA5 as a potential therapeutic target in esophageal squamous cell carcinoma. EBioMedicine.2019;46:54-65.

23. Fu G, Xu Z, Chen X, Pan H, Wang Y, Jin B. CDCA5 functions as a tumor promoter in bladder cancer by dysregulating mitochondria-mediated apoptosis, cell cycle regulation and $\mathrm{PI} / 3 \mathrm{k} / \mathrm{AKT} / \mathrm{mTOR}$ pathway activation. J Cancer.2020;11:2408-2420.

24. Jeffery J, Sinha D, Srihari S, Kalimutho M, Khanna KK. Beyond cytokinesis: the emerging roles of CEP55 in tumorigenesis. Oncogene.2016;35:683-690. 
25. Kalimutho M, Sinha D, Jeffery J, Nones K, Srihari S, Fernando WC, Duijf PH, Vennin C, Raninga P, Nanayakkara D et al. CEP55 is a determinant of cell fate during perturbed mitosis in breast cancer. EMBO Mol Med.2018;10.

26. Li M, Gao J, Li D, Yin Y. CEP55 Promotes Cell Motility via JAK2『STAT3هMMPs Cascade in Hepatocellular Carcinoma. Cells.2018;7.

27. Xu L, Xia C, Sheng F, Sun Q, Xiong J, Wang S. CEP55 promotes the proliferation and invasion of tumour cells via the AKT signalling pathway in osteosarcoma. Carcinogenesis.2018;39:623-631.

28. Tachiwana H, Müller S, Blümer J, Klare K, Musacchio A, Almouzni G. HJURP involvement in de novo CenH3(CENP-A) and CENP-C recruitment. Cell Rep.2015;11:22-32.

29. Chen T, Huang H, Zhou Y, Geng L, Shen T, Yin S, Zhou L, Zheng S. HJURP promotes hepatocellular carcinoma proliferation by destabilizing $\mathrm{p} 21$ via the MAPK/ERK1/2 and AKT/GSK3 $\beta$ signaling pathways. J Exp Clin Cancer Res.2018;37:193.

30. Chen T, Zhou L, Zhou Y, Zhou W, Huang H, Yin S, Xie H, Zhou L, Zheng S. HJURP Promotes Epithelialto-Mesenchymal Transition via Upregulating SPHK1 in Hepatocellular Carcinoma. Int J Biol Sci.2019;15:1139-1147.

31. Serafim RB, Cardoso C, Di Cristofaro LFM, Pienna Soares C, Araújo Silva W, Jr., Espreafico EM, PaçóLarson ML, Price BD, Valente V. HJURP knockdown disrupts clonogenic capacity and increases radiation-induced cell death of glioblastoma cells. Cancer Gene Ther.2020;27:319-329.

32. Wang CJ, Li X, Shi P, Ding HY, Liu YP, Li T, Lin PP, Wang YS, Zhang GQ, Cao Y. Holliday junction recognition protein promotes pancreatic cancer growth and metastasis via modulation of the MDM2/p53 signaling. Cell Death Dis.2020;11:386.

33. Lub S, Maes A, Maes K, De Veirman K, De Bruyne E, Menu E, Fostier K, Kassambara A, Moreaux J, Hose $D$ et al. Inhibiting the anaphase promoting complex/cyclosome induces a metaphase arrest and cell death in multiple myeloma cells. Oncotarget.2016;7:4062-4076.

34. Gu C, Yang Y, Sompallae R, Xu H, Tompkins VS, Holman C, Hose D, Goldschmidt H, Tricot G, Zhan F et al. FOXM1 is a therapeutic target for high-risk multiple myeloma. Leukemia.2016;30:873-882.

35. Liu X, Peng J, Zhou Y, Xie B, Wang J. Silencing RRM2 inhibits multiple myeloma by targeting the Wnt/ $\beta$-catenin signaling pathway. Mol Med Rep.2019;20:2159-2166.

36. de Boussac H, Bruyer A, Jourdan M, Maes A, Robert N, Gourzones C, Vincent L, Seckinger A, Cartron $\mathrm{G}$, Hose $\mathrm{D}$ et al. Kinome expression profiling to target new therapeutic avenues in multiple myeloma. Haematologica.2020;105:784-795.

37. Nara M, Teshima K, Watanabe A, Ito M, Iwamoto K, Kitabayashi A, Kume M, Hatano Y, Takahashi N, lida $S$ et al. Bortezomib reduces the tumorigenicity of multiple myeloma via downregulation of upregulated targets in clonogenic side population cells. PLoS One.2013;8:e56954.

38. Cohen Y, Gutwein O, Garach-Jehoshua O, Bar-Haim A, Kornberg A. The proliferation arrest of primary tumor cells out-of-niche is associated with widespread downregulation of mitotic and transcriptional genes. Hematology.2014;19:286-292. 


\section{Figures}
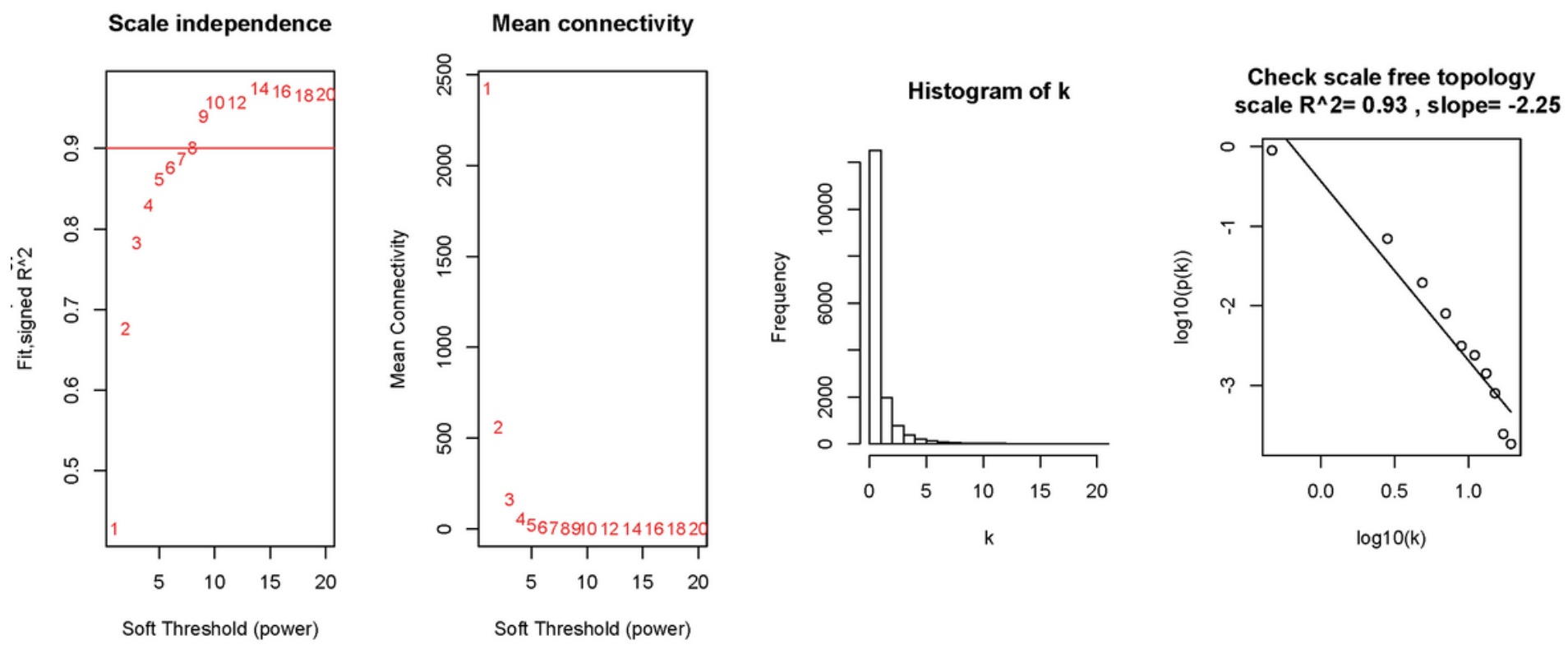

Figure 1

Determination of soft-thresholding power in weighted gene co-expression network analysis. (A) Analysis of scale-free fit index for different soft-thresholding powers $(\beta)$. Numbers in plots represent corresponding soft-thresholding powers. The approximate scale-free topology can be obtained at a soft-thresholding power of 9. Analysis of mean connectivity for different soft-thresholding powers. (B) Histogram of connectivity distribution when $\beta=9$. Checking scale-free topology when $\beta=9$. 


\section{Cluster Dendrogram}

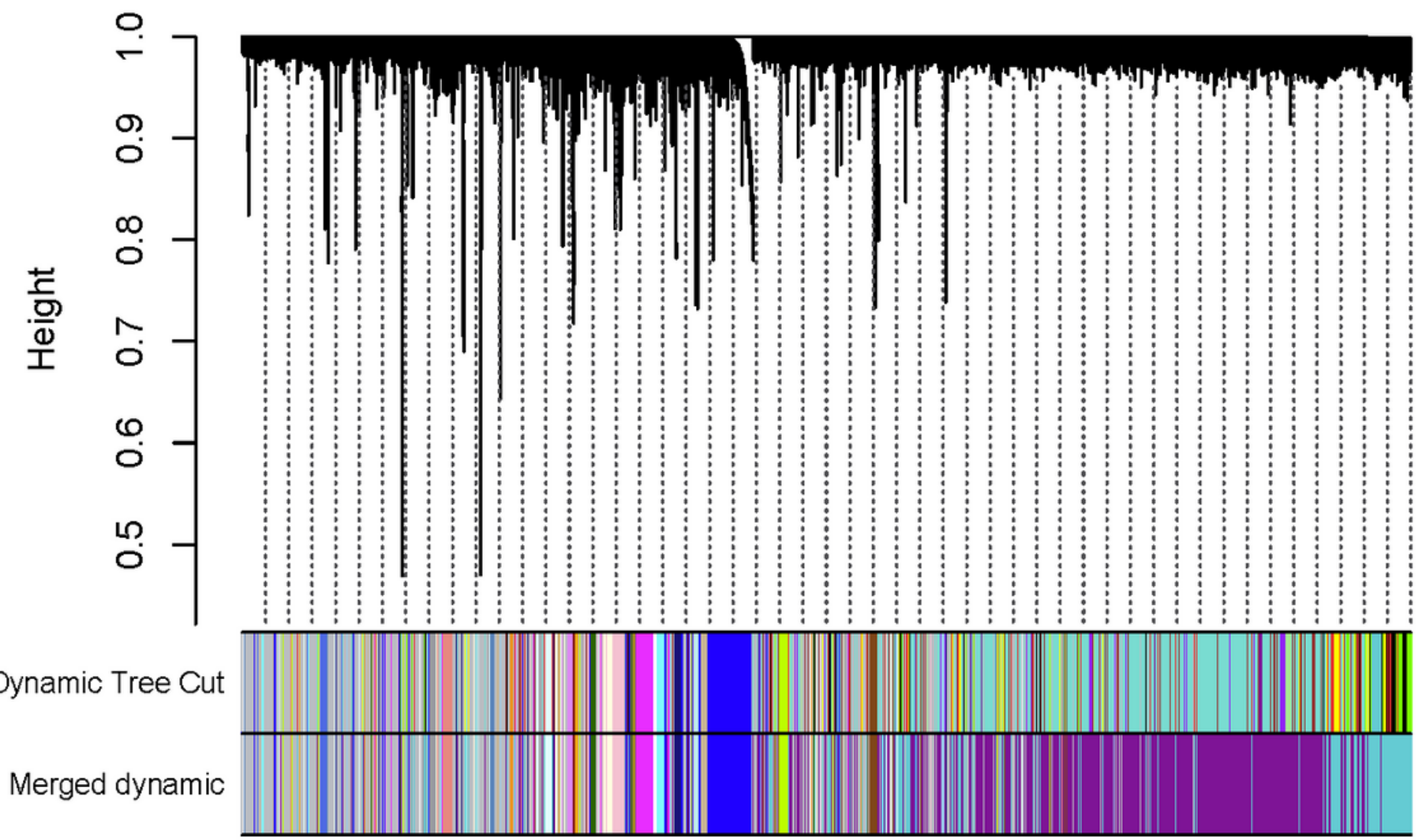

Figure 2

Dendrogram of modules identified by WGCNA. 


\section{Module-trait relationships}

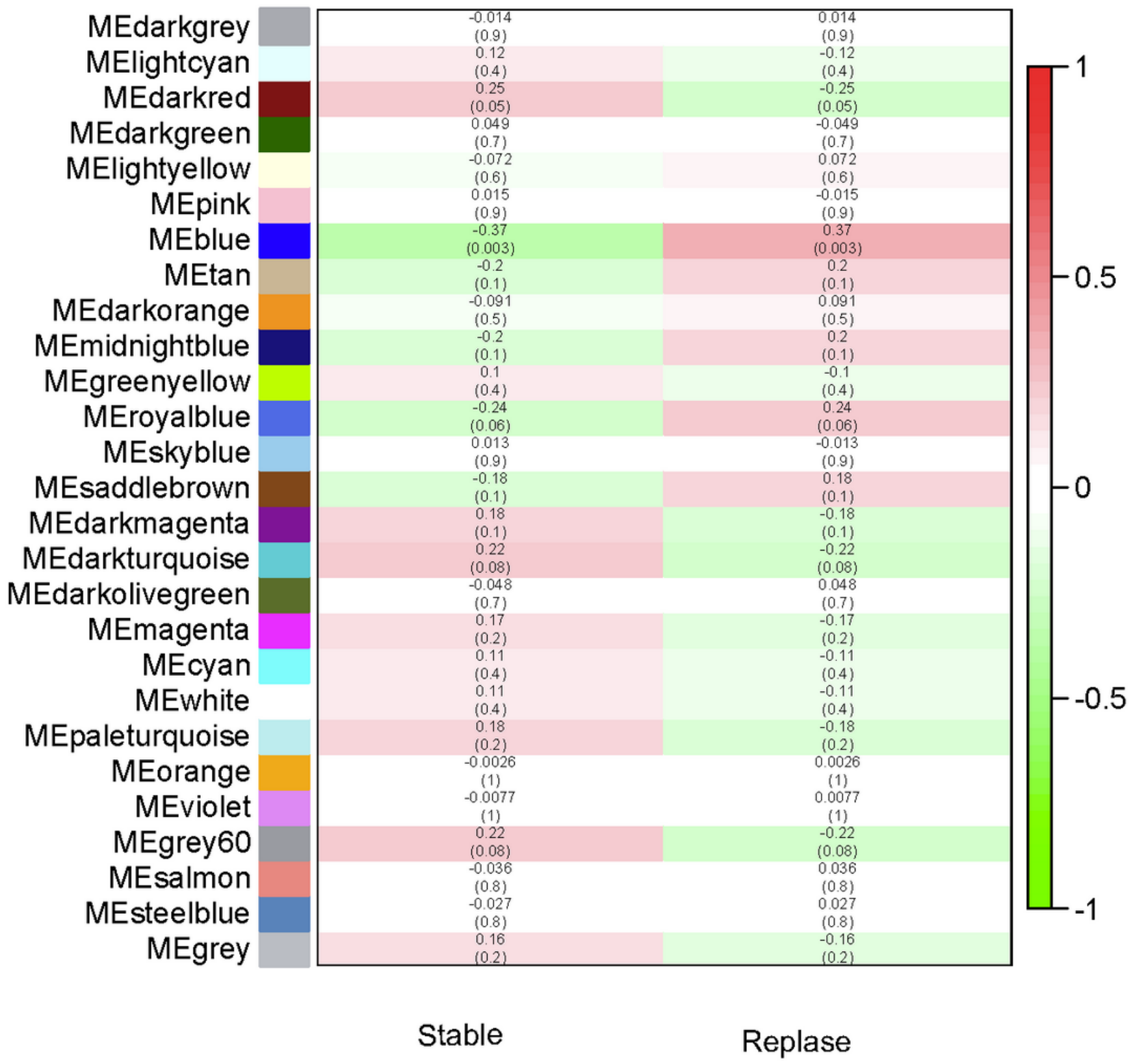

Figure 3

Heatmap of correlation between module genes and the therapeutic reaction of MM. Each module is based on the pattern of their co-expression. 


\section{Module membership vs. gene significance \\ cor=0.6, $\mathrm{p}=4 \mathrm{e}-102$}

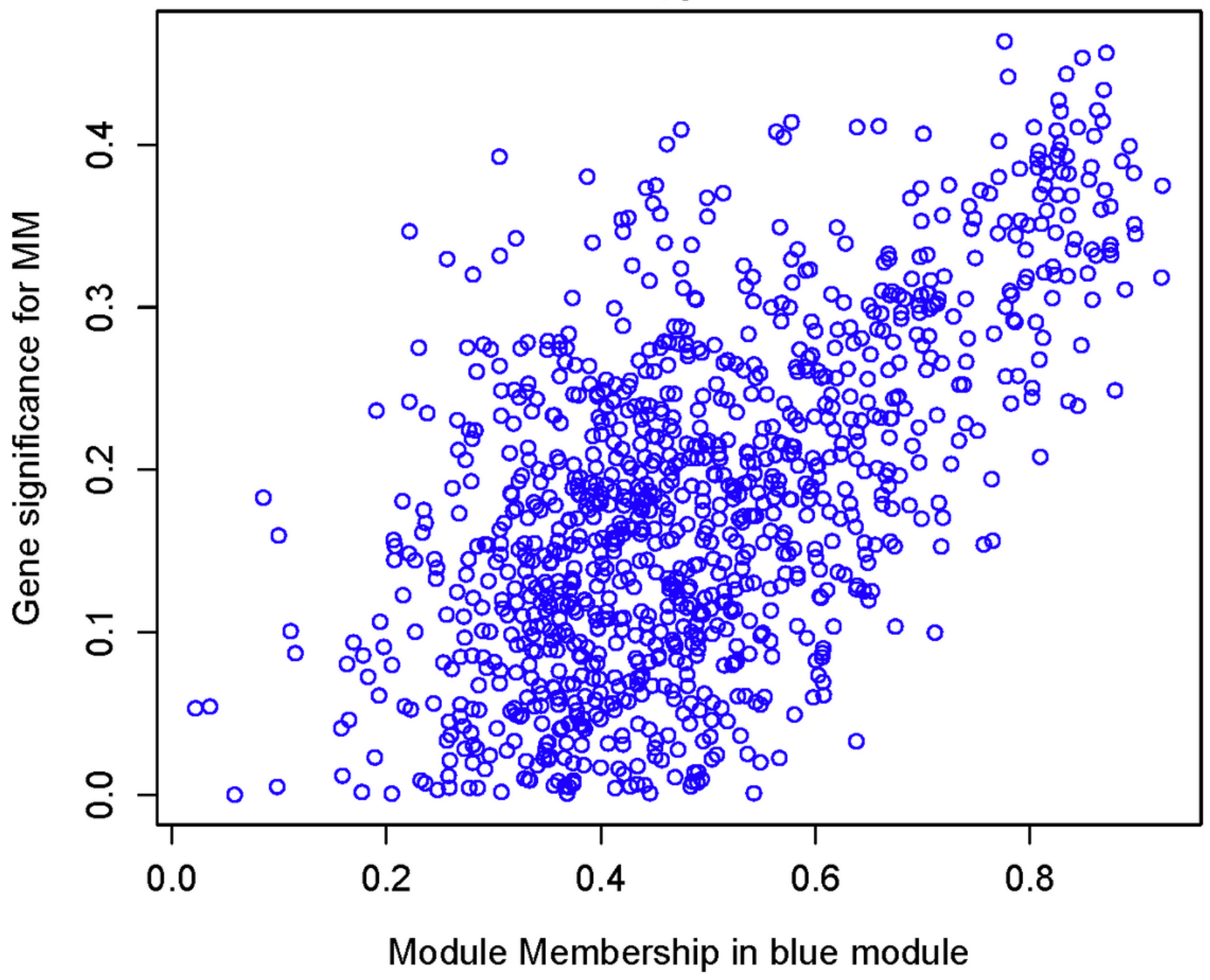

Figure 4

The scatter plot between blue module membership and gene significance for disease recurrence. 


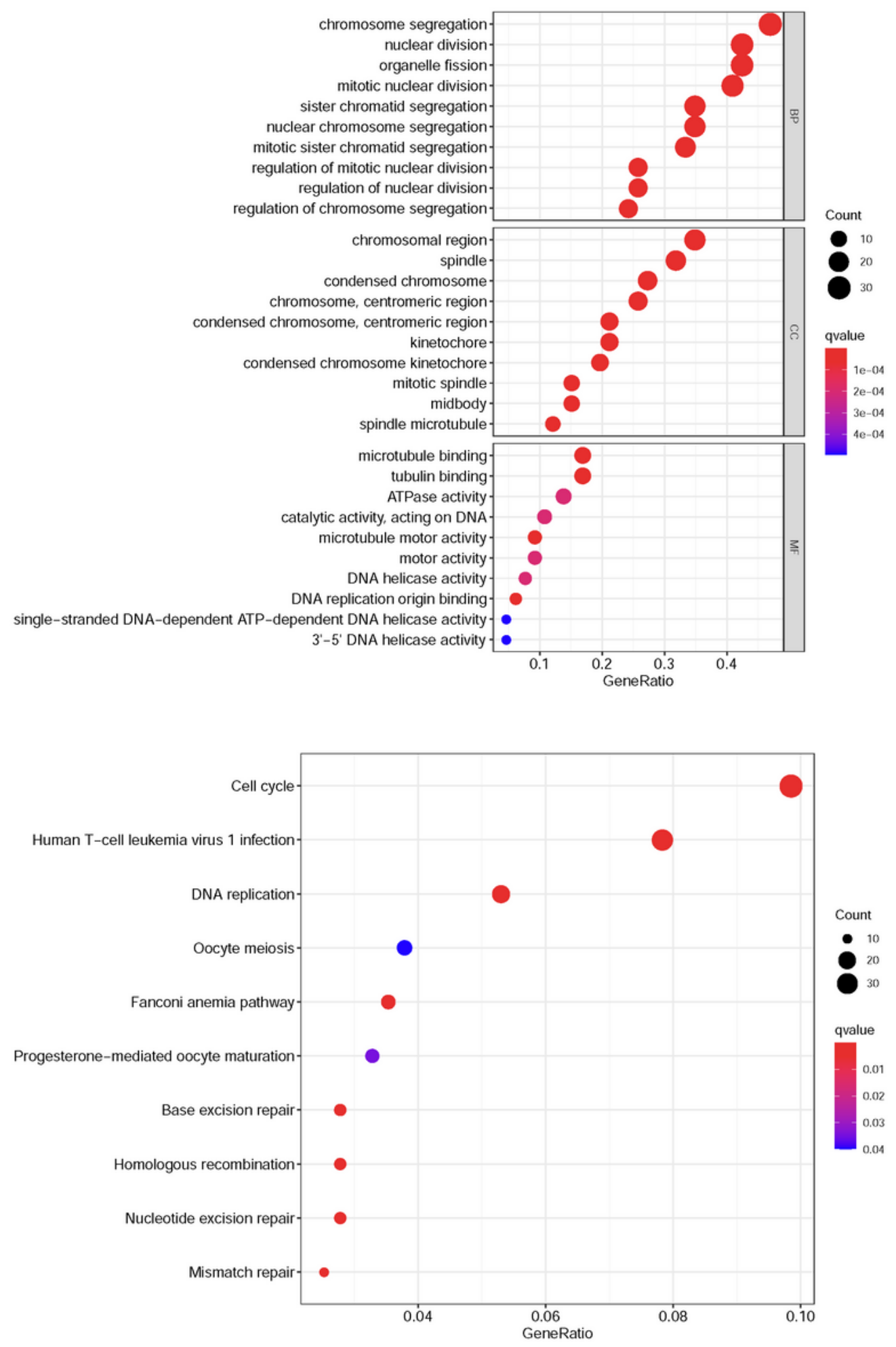

Figure 5

GO enrichment and KEGG pathway analysis in co-expression modules. (A): The results of BP, CC, MF in GO enrichment analysis. (B): The results of KEGG pathway analysis. 


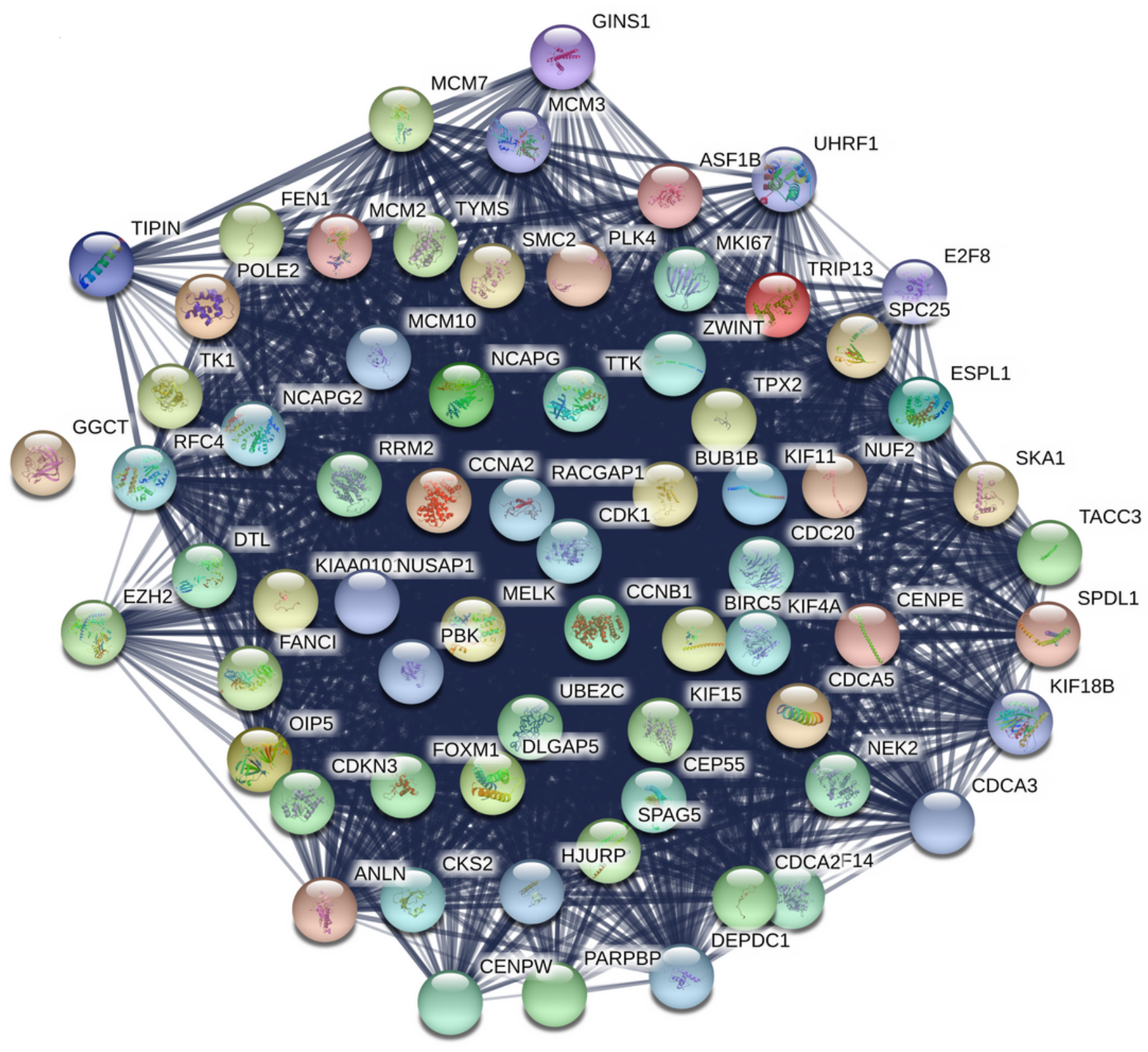

\section{Figure 6}

PPI network of 68 most connected genes. Nodes and lines represent genes and the correlation between genes. 

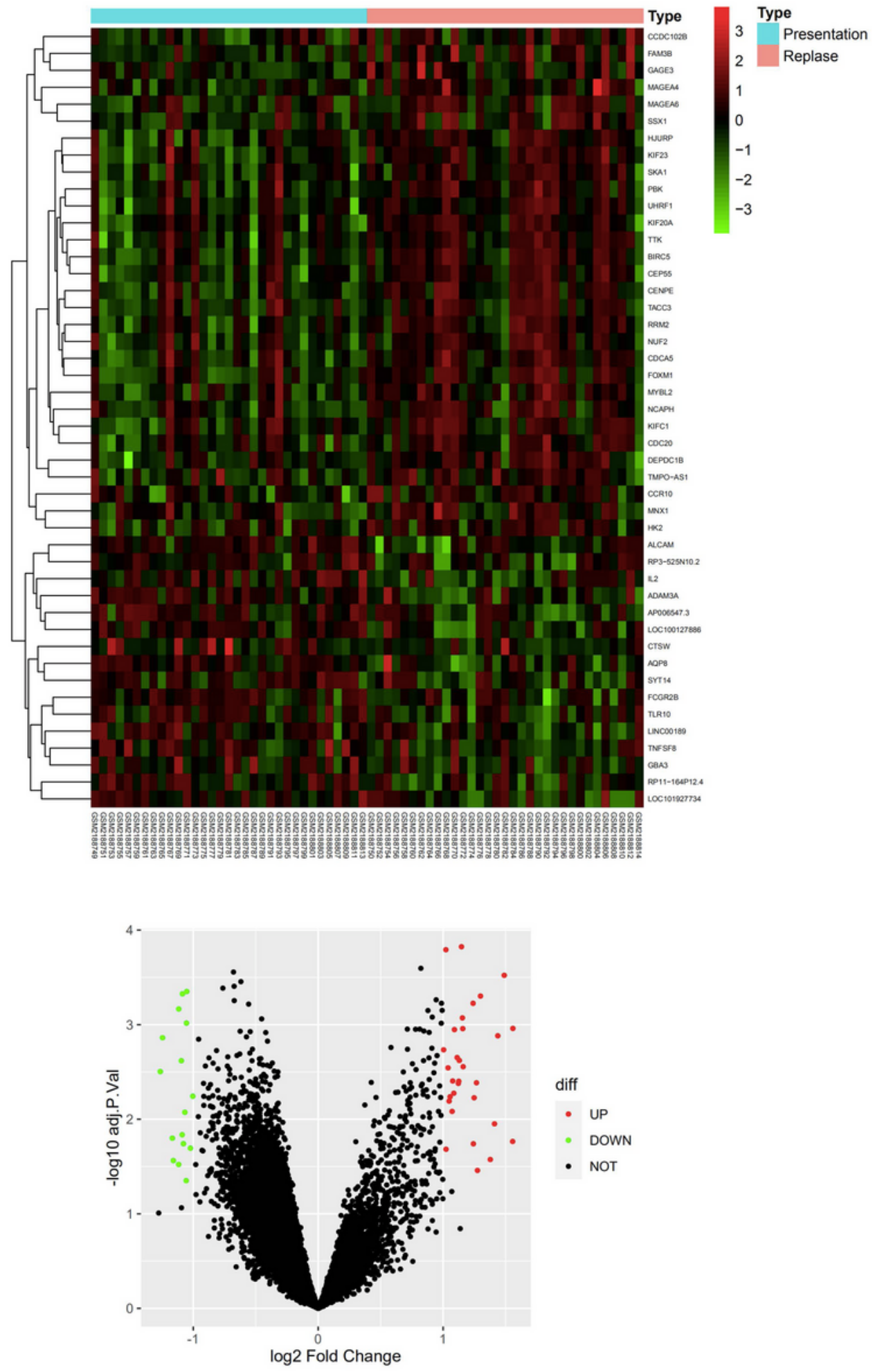

Figure 7

(A): Heatmap of all samples. The diagram presents the result of a two-way hierarchical clustering of all samples. (B):X-axis represents log2(Fold change) and Y-axis represents -log10(adj.P.Val). 


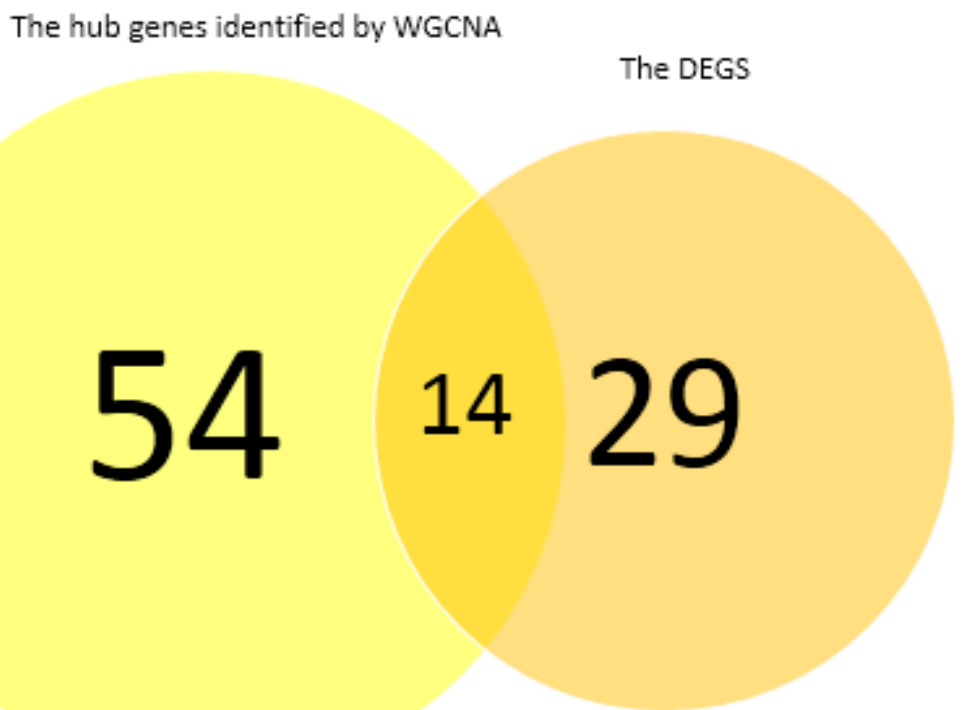

Figure 8

The Venn diagram of DEGs and hub genes. The overlapping area represents key genes. 

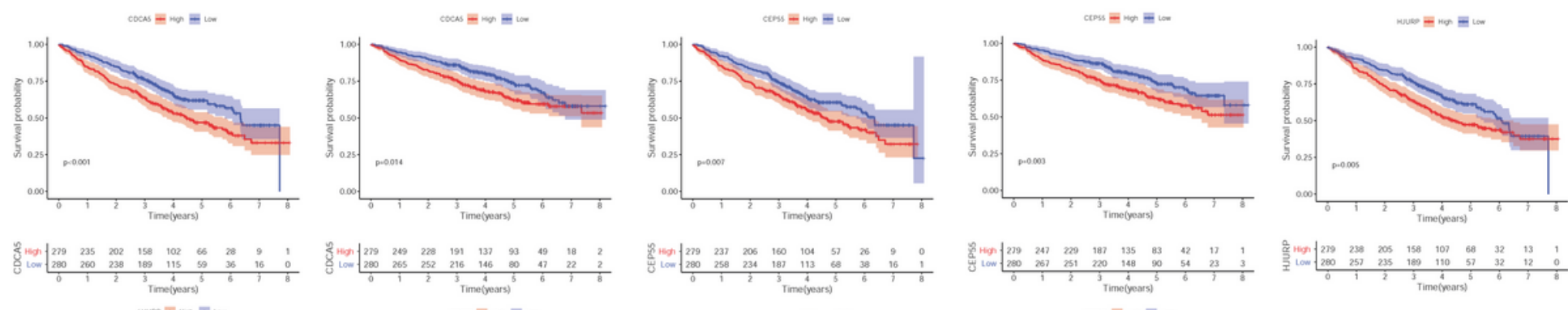

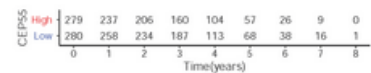

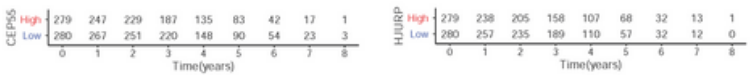
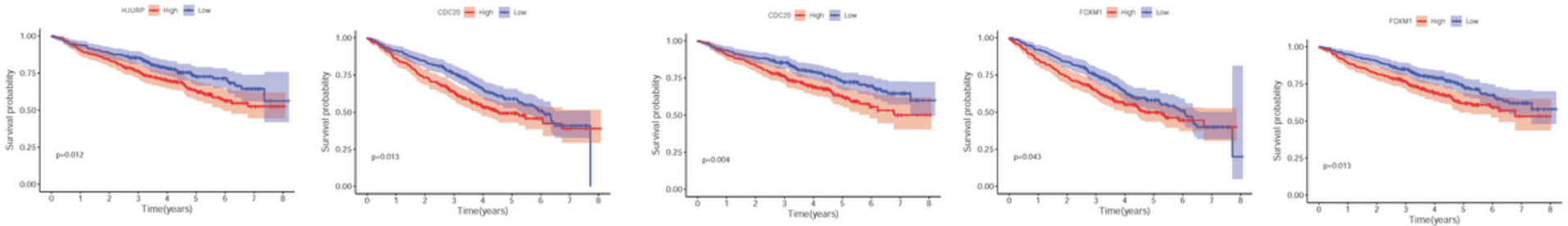

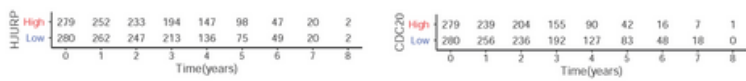

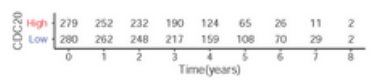

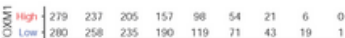
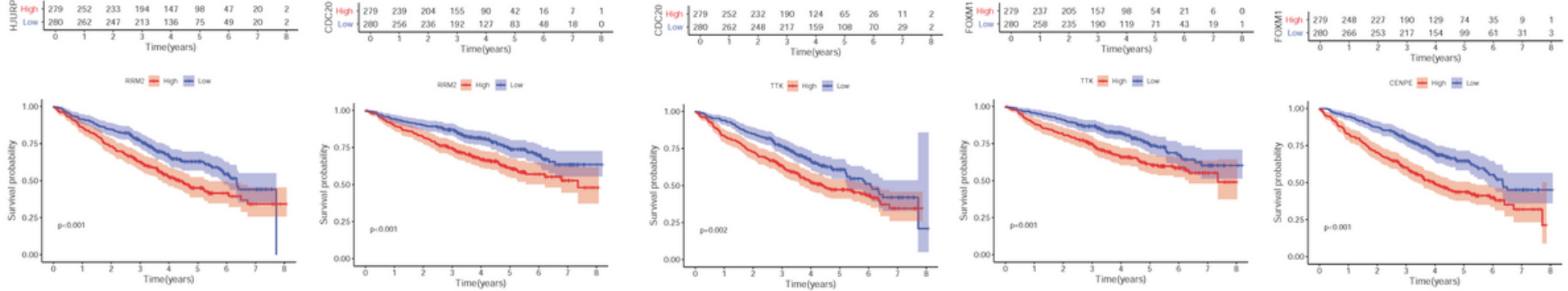

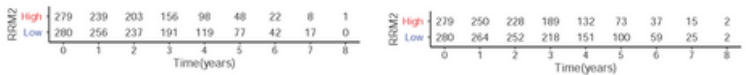

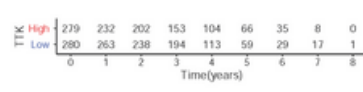

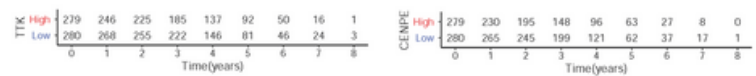
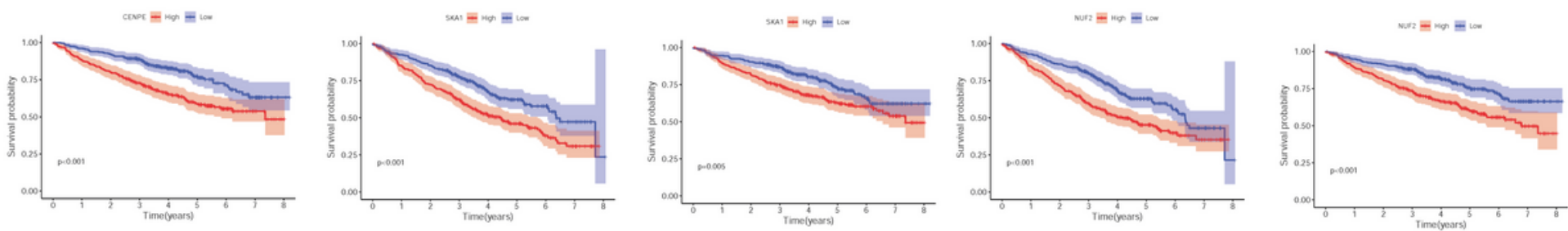

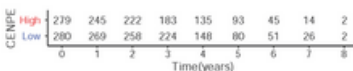

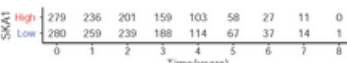

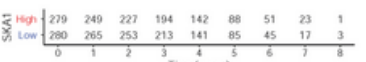

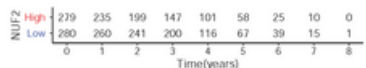

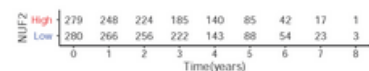

\section{Figure 9}

Survival analysis(EFS and OS, respectively) of key genes. 